\title{
EDITORIAL
}

\section{TAILORED EXERCISES PROGRAM - CONCEPT OF EXERCISES AS MEDICINE}

Exercises programs that are customized and tailored according to the need of the individual is the focus of various international organizations. Today the concept of long and continuous form of exercises has seen a paradigm shift towards the concept of exercises as medicine where health care professional are focusing towards designing and prescribing exercises that fits the need of individual and that could be discontinued once the desired goals are achieved. Evidences in favor of tailored exercises programs have further strengthen this concept where various literatures have suggested that customized and tailored exercise programs does not only provides short term benefits but certainly found to be helpful in improving agility, cardiovascular fitness, lung compliance, weight management and improving social life in long run as well.

Multiple evidences related to the effectiveness of prescribing exercises for achieving the short term and long term benefits in the management of various medical conditions has been well documented in numbers of literatures however very few literatures are available that have focused towards the concept of prescribing exercises in term of its dosage and have calculated the potency of particular exercise regime with respect to a particular program.

A very common problem that have been faced by the researcher to identify the dosage of exercises for the purpose of calculating its efficacy and cause effective relationship in response to the disease is the quantification of exercises. Previously exercises have been considered as a qualitative entity that was difficult to measured but with the advancement of research and evidence based practices various protocols and measures have been introduced for the purpose of the quantification of exercises that are workable not only for calculating the prescribed dosage but indeed are effective in measuring the potency of exercise

Researches in these days are required to conduct studies that not only focus towards identifying the effectiveness of exercises in response to the disease but must incorporate a strategy to calculate the dose response of exercises so that future studies and clinical practices could be tailored down up to the point where the maximum benefit can be achieved in a shorter duration of time that could turn to be a win- win situation for both the patient and the clinician.

Lastly the concept of dose response curve is required to be well understood before conducting the research on a large scale as plotting these curve required immense clerical work and recording of huge data that should be carefully published so that future studies with the same result could make the methodology and the result generalized at a larger scale.

\author{
Ali Farhad \\ Assistant Professor \\ Ziauddin College of Rehabilitation Sciences \\ Ziauddin University
}

